\title{
Minimally Invasive Transforaminal Lumbar Interbody Fusion: Comparison of Isthmic Versus Degenerative Spondylolisthesis
}

DUSTIN H. MASSEL, BS, BENJAMIN C. MAYO, BA, GRANT D. SHIFFLETT, MD, DANIEL D. BOHL, MD, MPH, PHILIP K. LOUIE, MD, BRYCE A. BASQUES, MD, WILLIAM W. LONG, BA, KRISHNA D. MODI, BS, FADY Y. HIJJI, BS, ANKUR S. NARAIN, BA, KERN SINGH, MD

Department of Orthopaedic Surgery, Rush University Medical Center, Chicago, Illinois

\begin{abstract}
Background: Minimally invasive transforaminal lumbar interbody fusion (MIS TLIF) is a common surgical procedure for treatment of degenerative spondylolisthesis (DS) but remains controversial for treatment of isthmic spondylolisthesis (IS). Few studies have compared IS and DS outcomes after MIS TLIF. Therefore, the objective of the current study was to compare outcomes of patients with IS and DS after MIS TLIF.

Methods: A retrospective cohort analysis was performed on a prospectively maintained database of patients who underwent a primary, 1-level MIS TLIF for grade I or II IS or DS. Grade I and II DS and grade I IS patients were treated with MIS TLIF via a unilateral tubular approach, whereas the grade II IS patients were treated via a bilateral tubular approach. Differences in patient demographics and preoperative characteristics were assessed using independent sample $t$ tests and $\chi^{2}$ tests. The type of spondylolisthesis and its effect on postoperative outcomes was analyzed using Poisson regression with robust error variance (binary outcomes) or linear regression (continuous outcomes) adjusted for preoperative characteristics. Subgroup analysis comparing grade I IS versus DS and grade II IS versus DS was performed.

Results: A total of 223 patients were included (IS: 62 [27.8\%]; DS: 161 [72.2\%]). IS patients were younger $(P<$ $.001)$, had a lower comorbidity burden $(P<.001)$, and a greater incidence of grade II spondylolisthesis $(P<.001)$ at L5-S1 $(P<.001)$ than the DS cohort. Patients with IS experienced longer operative times $(P<.001)$ and lower, but not statistically significant, arthrodesis rates compared to the DS cohort. No differences were observed in the remaining preoperative patient characteristics, perioperative or postoperative outcomes.

Conclusions: Despite being younger and having a lower comorbidity burden than the DS cohort, similar outcomes were observed after MIS TLIF for IS patients.

Level of Evidence: 3.

Clinical Relevance: These results suggest MIS TLIF is an appropriate treatment option for IS patients despite the increased instability inherent with IS.

Minimally Invasive Surgery

Keywords: minimally invasive spine surgery, transforaminal lumbar interbody fusion, degenerative spondylolisthesis, isthmic spondylolisthesis, unilateral interbody cage, bilateral interbody cage, low-grade spondylolisthesis
\end{abstract}

\section{INTRODUCTION}

Spondylolisthesis is a common pathologic disorder of the spine defined by anterolisthesis of the cranial over the caudal vertebra. Several types of spondylolisthesis exist; however, the 2 most commonly observed forms are degenerative (DS) and isthmic (IS) spondylolisthesis (Figure 1). ${ }^{1}$ Occurring most often at L4-L5 in elderly females, DS is the result of intervertebral disc degeneration and arthritic changes to the facet joints resulting in segmental instability and subsequent anterolisthetic progression. This pathology commonly presents in the fourth and fifth decades of life. ${ }^{1-4}$ In contrast, IS most commonly occurs in healthy adolescent and young adult athletes as the result of repetitive, hyperextension trauma to the posterior elements. This often results in an acquired bilateral defect, or spondylolysis, of the pars interarticularis at L5 and anterolisthetic progression of L5 over S1. ${ }^{5,6}$ In conjunction with the intervertebral discs, the main function of the facet joints are to counteract anterior shear and torsion forces at their specific vertebral segment, acting as static stabilizers. Due to 


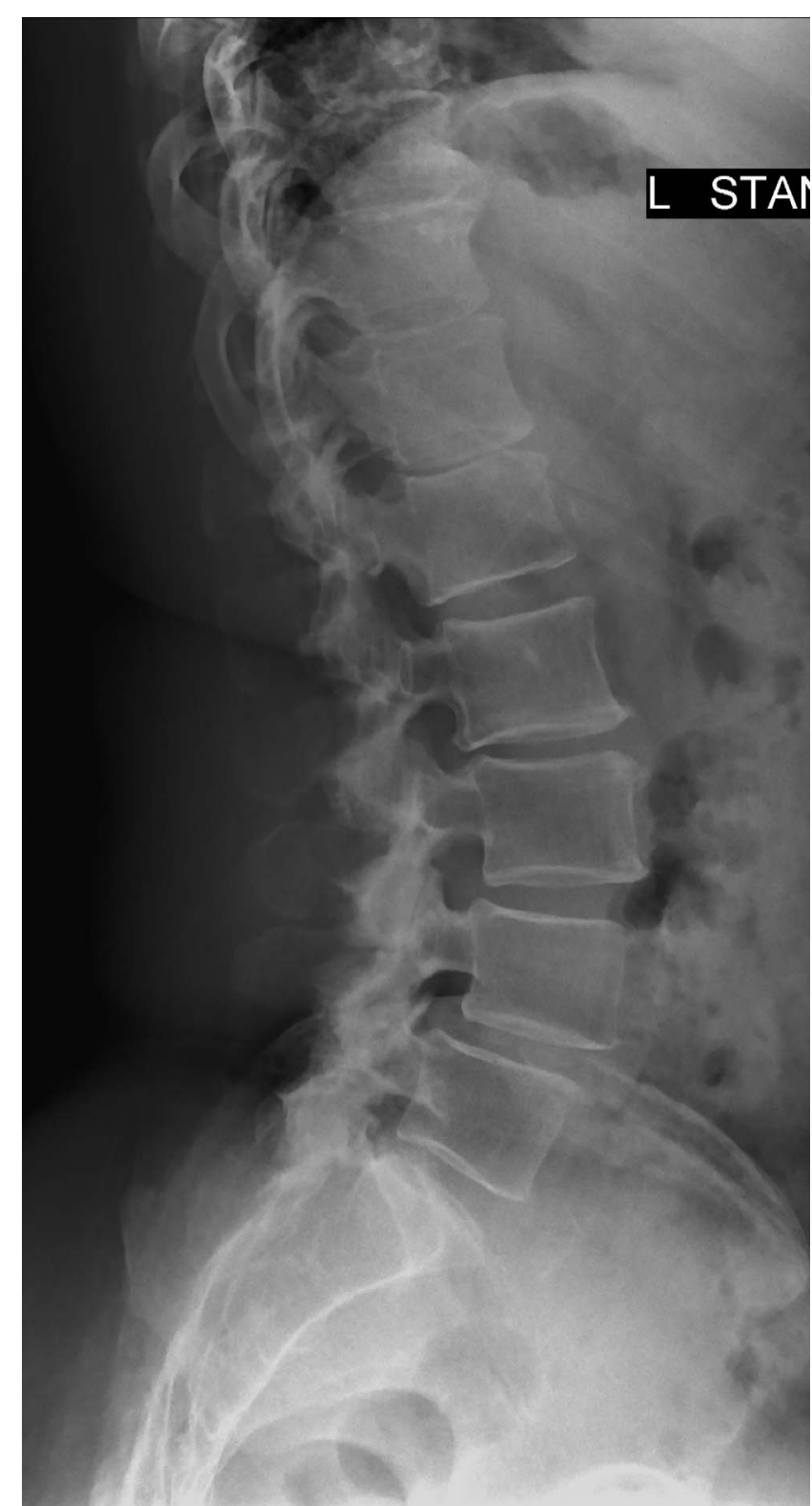

Figure 1. Preoperative radiographs demonstrating degenerative spondylolisthesis at L4-L5.

the interruption of the posterior elements, a lowgrade IS patient has an inherent instability that may result in greater anterolisthetic progression compared to a low-grade DS patient. $^{7-10}$

Often managed conservatively, a patient may experience refractory symptoms and elect to undergo surgical treatment, which often requires fusion surgery for definitive management. This can be accomplished in a variety of ways, one of which is minimally invasive (MIS) transforaminal lumbar interbody fusion (TLIF). Despite an initial surgical learning curve, MIS TLIF has enabled surgeons to perform fusion surgery with a reduction in incision size, iatrogenic soft tissue injury, operative time, estimated blood loss (EBL), postoperative pain, narcotic consumption, and perioperative complication rates compared to the open counterpart. ${ }^{11-17}$ The advantages afforded by the MIS TLIF technique have allowed a shift in surgical paradigm for several pathologic processes formerly treated via the open technique and have resulted in faster recovery time and a decreased length of hospitalization, among others. ${ }^{18-21}$

Several studies have examined the use of the MIS TLIF technique on low-grade DS (Meyerding grades I and II); however, the best approach for the surgical management of low-grade IS has not been fully elucidated. ${ }^{3}$ Similarly, few studies have compared the surgical outcomes of MIS TLIF between low-grade IS and DS. These studies are limited by underpowered patient populations, composed mainly of grade I spondylolisthesis patients. $^{22-27}$ Therefore, the purpose of this study was to compare clinical outcomes of patients with IS and DS after MIS TLIF in the largest comparative study of its kind.

\section{MATERIALS AND METHODS}

\section{Patient Population}

After institutional review board approval (ORA\#14051301), a prospectively maintained surgical database at a single academic institution was retrospectively reviewed. Patients who underwent a primary single-level MIS TLIF for the treatment of IS and DS between 2007 and 2015 were identified. Patients were excluded from the analysis if they radiographically demonstrated a spondylolisthesis grade III or above, were diagnosed with both IS and DS, or had less than 1 year of postoperative follow up.

\section{Demographic and Outcome Analysis}

Patients were stratified into either the IS or DS cohort (Figure 1) based on their presenting diagnosis. Patients were then analyzed by the following demographic, comorbidity, and perioperative variables: age, gender, body mass index (BMI, nonobese $[\mathrm{BMI}<30]$, obese [BMI $\geq 30]$ ), smoking status, and Charlson Comorbidity Index (CCI). Perioperative variables included spondylolisthesis level and grade, operative time, EBL, length of hospital stay (LOS), complication, arthrodesis, and revision rates, as well as the preoperative, postoperative, and change from 
preoperative to postoperative visual analogue scale (VAS) scores. Computed tomography (CT) scan was used to determine arthrodesis, defined by the presence of bony bridging on 3 sequential cuts in the sagittal and coronal planes. The presence of subchondral cysts, end plate sclerosis, or haloing around the interbody cage(s) or pedicle screws were also evaluated for possible pseudarthrosis. It is standard practice for the senior surgeon to obtain postoperative CT scans at the 6-month postoperative visit after all TLIFs. Additional CT scans are obtained at the 1-year postoperative visit if the patient underwent revision procedure, demonstrated incomplete fusion on the 6-month CT scan, or remained symptomatic.

\section{Surgical Technique}

Surgical treatment was based on the unique pathomechanical differences between IS and DS as well as the increasing degree of instability associated with progressively worsening grades of listhesis. ${ }^{28}$ Grade I and II DS and grade I IS patients were treated with MIS TLIF via a unilateral approach. Grade II IS patients were treated with a bilateral tubular approach. After paraspinal skin incision, the primary approach was obtained using the Wiltse technique under fluoroscopic guidance. For patients with either grade I or II DS, unilateral laminectomy and facetectomy were performed through a single 21-mm nonexpandable tubular retractor. For patients with grade I IS, unilateral laminectomy and facetectomy were performed, and for those with grade II IS, bilateral laminectomy and facetectomy were performed through bilateral 21-mm nonexpandable tubular retractors. After decompression, the intervertebral disc was identified, incised and removed; the end plates and bone graft were prepared. A mixture of autograft obtained from the laminectomy and facetectomy was morselized and mixed with $5 \mathrm{cc}$ of bone marrow aspirate from the cannulated pedicles and $15 \mathrm{cc}$ of allograft cancellous bone. This bone graft mixture was impacted into the front of the disc space before interbody cage placement. Additionally, the single interbody cage was prepared with an extra small kit rhBMP-2 (2.1 mg), and the bilateral interbody cages were prepared with a small kit rhBMP-2 $(4.2 \mathrm{mg})$ divided between the 2 cages. A single interbody cage was impacted into place for grade I IS and grades I and II DS cohorts and bilateral interbody cages for grade II IS (Figures 2A and B). Although placement of bilateral interbody devices is often associated with a posterior lumbar interbody fusion, the procedure involved bilateral laminectomies and facetectomies allowing for a transforaminal approach through Kambin's triangle. Percutaneous pedicle screws were placed bilaterally over guide wires. No posterolateral fusion was performed, and preservation of the midline musculature and ligamentous structures was achieved. Of note, rhBMP-2 was used as an off-label bone graft substitute in all MIS TLIFs performed.

\section{Statistical Methods}

Statistical analysis was performed using Stata/ MP 13.1 for Mac (StataCorp LP, College Station, TX). Differences between patients in terms of demographics, comorbidities, and procedural characteristics were tested for using Pearson's $\chi^{2}$ test (for categorical characteristics) and independent sample $t$ tests (for continuous characteristics). Multivariate linear regression (for continuous variables) and Poisson regression with robust error variance (for binary outcomes) were used to test for differences between IS and DS patients with respect to procedural variables (operative time, EBL, LOS, postoperative VAS, change in VAS) and postoperative outcomes (complication, arthrodesis, and revision rates). ${ }^{29}$ These regressions were each adjusted for baseline patient characteristics. Each of these analyses were performed when first comparing IS and DS as well as within the subgroup analyses between grade I IS and DS and grade II IS and DS. A $P$ value $<.05$ was used to determine statistical significance.

\section{RESULTS \\ Primary Analysis}

A total of 223 patients were identified, of which $62(27.8 \%)$ were diagnosed with IS and $161(72.2 \%)$ were diagnosed with DS. Baseline characteristics are detailed in Table 1. The IS cohort was younger (47.3 \pm 13.9 versus $56.9 \pm 11.2$ years, $P<.001)$, had a lower comorbidity burden $(2.2 \pm 2.0$ versus $3.6 \pm$ $2.2, P<.001)$, and had a greater incidence of grade II spondylolisthesis $(64.5 \%$ versus $7.5 \%, P<.001)$ at L5-S1 $(71.0 \%$ versus $17.4 \%, P<.001)$ than the DS cohort. There were no significant differences in gender, body mass index, smoking status, or preoperative VAS pain scores between the 2 cohorts $(P>.05)$. 


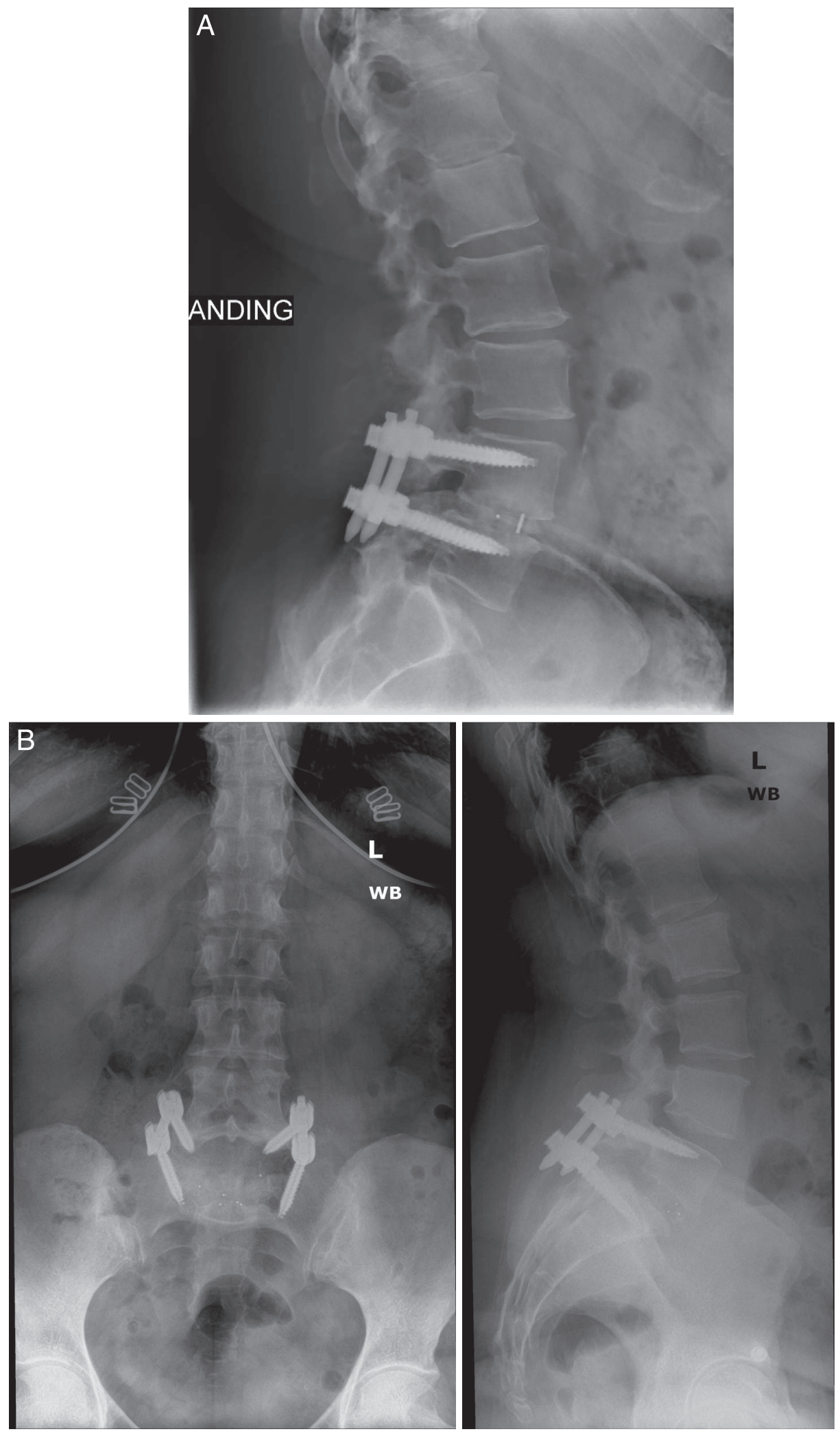

Figure 2. Postoperative radiographs demonstrating (A) a unilateral interbody cage in a patient with grade I degenerative spondylolisthesis and (B) a bilateral interbody cage in a patient with grade II isthmic spondylolisthesis. 
Table 1. Baseline characteristics. ${ }^{a}$

\begin{tabular}{|c|c|c|c|}
\hline & \multicolumn{2}{|c|}{ Spondylolisthesis $^{\mathrm{b}}$} & \multirow[b]{2}{*}{$P$ Value } \\
\hline & Isthmic $(\mathrm{N}=62)$ & Degenerative $(\mathrm{N}=161)$ & \\
\hline Age $($ mean $\pm \mathrm{SD}, \mathrm{y})$ & $47.3 \pm 13.9$ & $56.9 \pm 11.2$ & $<.001$ \\
\hline Sex, \% (n) & & & .323 \\
\hline Female & $43.6(27)$ & $50.9(82)$ & \\
\hline Male & $56.5(35)$ & $49.1(79)$ & \\
\hline BMI, \% (n) & & & .555 \\
\hline Nonobese (BMI < 30) & $48.4(30)$ & $52.8(85)$ & \\
\hline Obese $(\mathrm{BMI} \geq 30)$ & $51.6(32)$ & $47.2(76)$ & \\
\hline Smoking status, \% (n) & & & .212 \\
\hline Nonsmoker & $75.8(47)$ & $83.1(133)$ & \\
\hline Smoker & $24.2(15)$ & $16.9(27)$ & \\
\hline Level of Spondylolisthesis, \% (n) & & & $<.001$ \\
\hline L3-L4 & $0.0(0)$ & $7.5(12)$ & \\
\hline L4-L5 & $29.0(18)$ & $75.2(121)$ & \\
\hline L5-S1 & $71.0(44)$ & $17.4(28)$ & \\
\hline Spondylolisthesis grade, \% (n) & & & $<.001$ \\
\hline Grade I & $35.5(22)$ & $92.6(149)$ & \\
\hline Grade II & $64.5(40)$ & $7.5(12)$ & \\
\hline Comorbidity burden $(\mathrm{CCI})$ & $2.2 \pm 2.0$ & $3.6 \pm 2.2$ & $<.001$ \\
\hline Preoperative VAS, mean $\pm \mathrm{SD}$, min & $7.2 \pm 1.8$ & $7.0 \pm 2.0$ & .405 \\
\hline
\end{tabular}

Abbreviations: BMI, body mass index; CCI, Charlson comorbidity index; SD, standard deviation; VAS, visual analogue scale.

${ }^{a}$ Boldface indicates statistical significance.

${ }^{\mathrm{b}}$ Three patients were excluded due to having both isthmic and degenerative spondylolisthesis.

Table 2 contains perioperative and postoperative outcome comparisons. Patients with IS experienced longer operative times (146.6 \pm 44.6 versus $122.6 \pm$ $41.0 \mathrm{~min}, P<.001)$ compared to the DS cohort. The remainder of the perioperative and postoperative outcomes were similar between the IS and DS cohorts. These variables include: LOS; postoperative VAS pain scores at 6 weeks, 12 weeks, and 6 months; change in VAS from preoperative to postoperative time points; complication; arthrodesis (Figures $3 \mathrm{~A}$ and $\mathrm{B}$ ); and revision rates. Of note, a 73-year-old female experienced motor and sensory neuropraxia after her primary procedure. Weakened dorsiflexion and left dorsal foot numbness correlated with a reduced compound muscle action potential amplitude in the left common peroneal nerve on electromyography (EMG). The patient underwent a revision decompression for recurrent lateral stenosis

Table 2. Outcomes. ${ }^{a}$

\begin{tabular}{|c|c|c|c|}
\hline & \multicolumn{2}{|c|}{ Spondylolisthesis $^{\mathbf{b}}$} & \multirow[b]{2}{*}{$P$ Value $^{\mathrm{c}}$} \\
\hline & Isthmic $(\mathrm{N}=62)$ & Degenerative $(N=161)$ & \\
\hline Operative time, mean $\pm \mathrm{SD}$, min & $146.6 \pm 44.6$ & $122.6 \pm 41.0$ & .003 \\
\hline Estimated blood loss, mL & $97.7 \pm 103.3$ & $67.8 \pm 59.5$ & .101 \\
\hline Length of hospital stay, h & $68.5 \pm 52.5$ & $69.7 \pm 45.6$ & .872 \\
\hline \multicolumn{4}{|l|}{$\mathrm{VAS}$, mean $\pm \mathrm{SD}$} \\
\hline 6-wk VAS & $4.2 \pm 2.2$ & $3.6 \pm 2.2$ & .986 \\
\hline 12-wk VAS & $3.7 \pm 2.0$ & $3.4 \pm 2.3$ & .888 \\
\hline 6-mo VAS & $3.9 \pm 2.5$ & $3.1 \pm 2.5$ & .945 \\
\hline \multicolumn{4}{|l|}{ Change in VAS, mean $\pm \mathrm{SD}^{\mathrm{d}}$} \\
\hline$\Delta$ VAS at $6 \mathrm{wk}$ & $-2.5 \pm 2.9$ & $-3.2 \pm 3.1$ & .941 \\
\hline$\Delta \mathrm{VAS}$ at $12 \mathrm{wk}$ & $-3.7 \pm 3.3$ & $-3.5 \pm 3.0$ & .888 \\
\hline$\Delta \mathrm{VAS}$ at $6 \mathrm{mo}$ & $-4.1 \pm 3.2$ & $-4.0 \pm 3.2$ & .446 \\
\hline Complications, \% (n) ${ }^{\mathrm{e}}$ & $1.6(1)$ & $2.5(4)$ & ${ }_{-}^{\mathrm{f}}$ \\
\hline Arthrodesis at $1 \mathrm{y}, \%(\mathrm{n})$ & $88.7(55)$ & 97.5 (157) & .864 \\
\hline Revision, \% (n $)^{\mathrm{g}}$ & $9.7(6)$ & $8.1(13)$ & .135 \\
\hline
\end{tabular}

Abbreviation: SD, standard deviation; VAS, visual analogue scale.

${ }^{a}$ Boldface indicates statistical significance.

${ }^{\mathrm{b}}$ Three patients were excluded due to having both isthmic and degenerative spondylolisthesis.

${ }^{\mathrm{c}} P$ value is from Poisson regression with robust error variance (binary outcomes) or linear regression (continuous outcomes) adjusted for age, sex, body mass index, operative level (L5-S1 versus other), smoking status, comorbidity burden, and preoperative VAS.

${ }^{\mathrm{d}}$ Change in VAS (for each patient) = postoperative VAS $(6 \mathrm{wk}, 12 \mathrm{wk}, 6 \mathrm{mo})-$ preoperative VAS.

${ }^{\mathrm{e}}$ Complications include pseudobowel obstruction requiring rectal tube placement, epidural hematoma or fluid collection requiring irrigation and debridement (3), thromboembolic event (deep vein thrombosis [2]).

${ }^{\mathrm{f}}$ Statistical analysis was not performed for variables with a total of $\leq 5$ occurrences

${ }^{\mathrm{g}}$ Revisions include pseudarthrosis (11), failed spinal instrumentation, recurrent symptoms requiring revision laminectomy (4), epidural hematoma or fluid collection requiring irrigation and debridement (3), recurrent stenosis with radiculopathy and foot drop. 

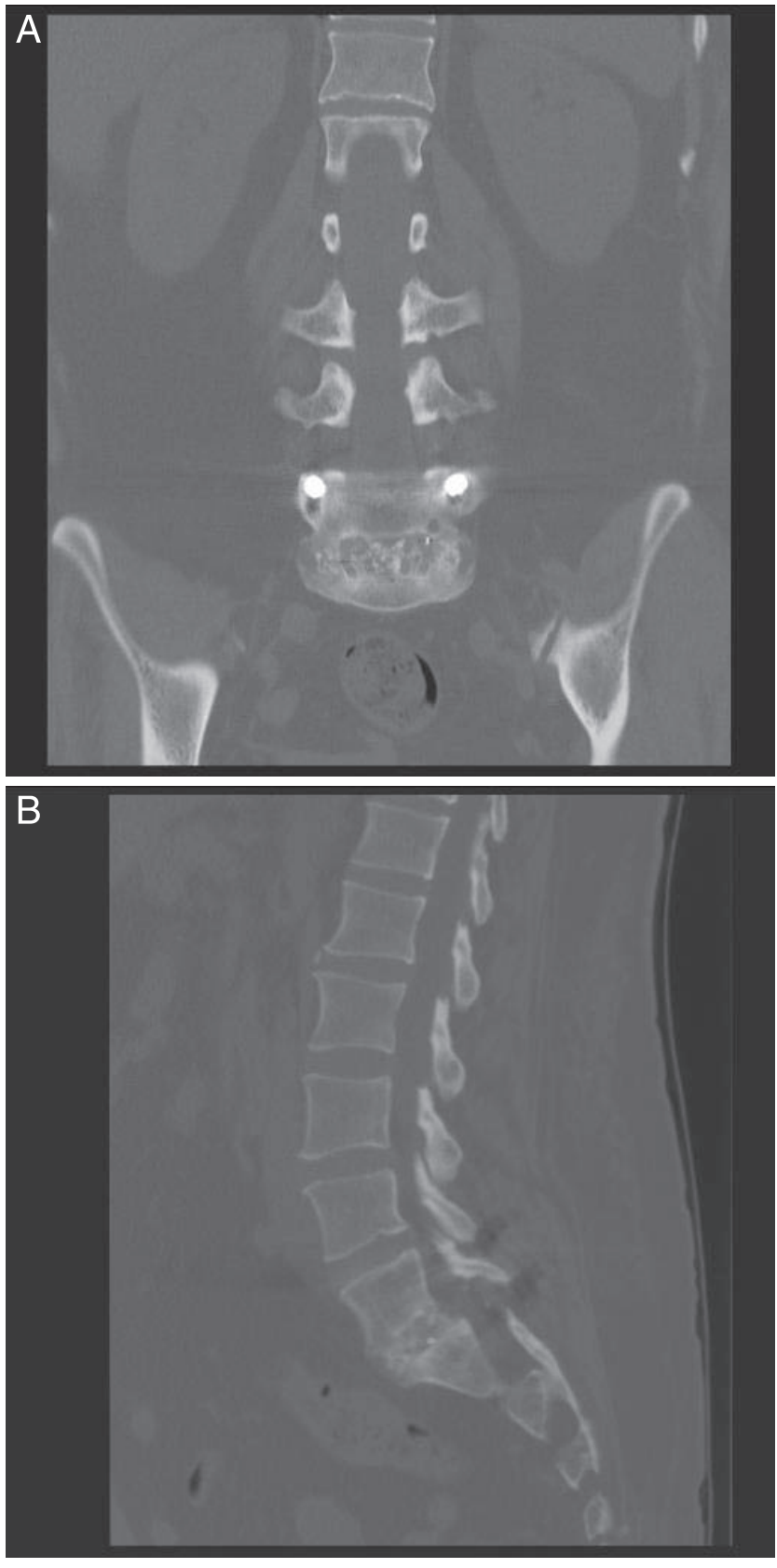

Figure 3. Postoperative (A) coronal and (B) sagittal computed tomographic scans demonstrating a bilateral interbody cage in a patient with grade II isthmic spondylolisthesis.

at L4-L5, left lower extremity radiculopathy, and dorsiflexion weakness. After revision operation, the patient was reevaluated with EMG and noted to have denervation changes in the peroneal muscle, distal to the fibular head. Improved dorsiflexion strength was noted by 18 months postoperatively.

Of the 62 patients diagnosed with IS, $22(35.5 \%)$ and $40(64.5 \%)$ were radiographically diagnosed with grade I and grade II IS, respectively. Of the 161 patients with DS, $149(92.5 \%)$ and $12(7.5 \%)$ were radiographically diagnosed with grade I and grade II DS, respectively.

\section{Subgroup Analysis}

Subgroup analysis was performed comparing the grade I IS and DS subgroups as well as grade II IS and DS subgroups. The grade I IS cohort was younger $(P<.001)$, had a lower comorbidity burden $(P=.003)$, a greater incidence of spondylolisthesis at the L5-S1 vertebral level $(P<.001$; Table 3), and experienced longer operative times $(P$ $=.030$ ) than the grade I DS cohort (Table 4). The remainder of the perioperative and postoperative outcomes did not differ between cohorts (Table 4).

The grade II IS cohort was younger $(P=.001)$, had a lower comorbidity burden $(P<.001)$, a greater incidence of spondylolisthesis at the L5-S1 vertebral level $(P<.001$; Table 3$)$, and experienced longer operative times $(P=.011)$ than the grade II DS cohort (Table 4). The remainder of the perioperative and postoperative outcomes did not differ between cohorts (Table 4).

\section{DISCUSSION}

Surgical management of symptomatic low-grade spondylolisthesis using MIS TLIF is well established in the current literature..$^{4,21-26,30-33}$ While evidence supporting the use of MIS TLIF for treatment of low-grade DS is robust, literature regarding the treatment of IS remains controversial, and data comparing MIS TLIF for low-grade IS and DS are limited. ${ }^{4,22-25,32,34}$

The results of this study suggest that MIS TLIF is a safe and effective procedure when using a single interbody cage and a unilateral tubular approach for grade I IS and grades I or II DS patients. Additionally, using bilateral interbody cages for grade II IS results in equivalent surgical outcomes. IS patients were statistically younger, had a lower comorbidity burden, and experienced spondylolisthesis at a more caudal vertebral level when compared to the DS cohort, but the most significant difference was an increased operative time. There are many explanations for this observation. The removal of excess fibrous connective and chondroid tissues from the fracture site often increases the operative times when compared to the DS dissection. ${ }^{35}$ Further, with disruption of the posterior arch and increased slippage, the anatomic relationships become further distorted and more difficult to 
Table 3. Baseline characteristics of subgroup analyses. ${ }^{a}$

\begin{tabular}{|c|c|c|c|c|c|c|}
\hline & \multicolumn{2}{|c|}{ Grade I Spondylolisthesis ${ }^{b}$} & \multirow[b]{2}{*}{$P$ Value } & \multicolumn{2}{|c|}{ Grade II Spondylolisthesis ${ }^{\text {b }}$} & \multirow[b]{2}{*}{$P$ Value } \\
\hline & Isthmic $(\mathrm{N}=22)$ & Degenerative $(N=149)$ & & Isthmic $(N=40)$ & Degenerative $(\mathrm{N}=12)$ & \\
\hline Age, mean $\pm \mathrm{SD}, \mathrm{y}$ & $41.8 \pm 14.5$ & $56.3 \pm 11.2$ & $<.001$ & $50.4 \pm 12.7$ & $64.1 \pm 8.9$ & .001 \\
\hline Sex, \% (n) & & & .669 & & & .335 \\
\hline Female & $45.5(10)$ & $50.3(75)$ & & $42.5(17)$ & $58.3(7)$ & \\
\hline Male & $54.6(12)$ & $49.7(74)$ & & $57.5(23)$ & $41.7(5)$ & \\
\hline BMI, \% (n) & & & .316 & & & .722 \\
\hline Nonobese $(\mathrm{BMI}<30)$ & $40.9(9)$ & $52.4(78)$ & & $52.5(21)$ & $58.3(7)$ & \\
\hline Obese $(\mathrm{BMI} \geq 30)$ & $59.1(13)$ & $47.7(71)$ & & 47.5 (19) & $41.7(5)$ & \\
\hline Smoking status, \% (n) & & & .091 & & & .893 \\
\hline Nonsmoker & $68.2(15)$ & $83.2(124)$ & & $80.0(32)$ & $81.8(9)$ & \\
\hline Smoker & $31.8(7)$ & $16.8(25)$ & & $20.0(8)$ & $18.2(2)$ & \\
\hline Level of spondylolisthesis, \% (n) & & & $<.001$ & & & $<.001$ \\
\hline L3-L4 & $0.0(0)$ & $6.7(10)$ & & $0.0(0)$ & $16.7(2)$ & \\
\hline L4-L5 & $27.3(6)$ & $74.5(111)$ & & 30.0 (12) & $83.3(10)$ & \\
\hline $\mathrm{L} 5-\mathrm{S} 1$ & $72.7(16)$ & $18.8(28)$ & & $70.0(28)$ & $0.0(0)$ & \\
\hline Comorbidity burden $(\mathrm{CCI})$, mean $\pm \mathrm{SD}$ & $2.0 \pm 2.4$ & $3.6 \pm 2.2$ & .003 & $2.4 \pm 1.8$ & $4.8 \pm 2.8$ & $<.001$ \\
\hline Preoperative VAS, mean $\pm \mathrm{SD}$, min & $7.1 \pm 1.9$ & $6.9 \pm 2.0$ & .664 & $7.2 \pm 1.8$ & $6.8 \pm 2.3$ & .557 \\
\hline
\end{tabular}

Abbreviations: BMI, body mass index; CCI, Charlson comorbidity index; SD, standard deviation; VAS, visual analogue scale.

${ }^{a}$ Boldface indicates statistical significance.

${ }^{\mathrm{b}}$ Three patients were excluded due to having both isthmic and degenerative spondylolisthesis.

visualize through a tubular approach. Moreover, grade II and higher IS requires a more extensive decompression that involves bilateral tubular approaches, laminectomies, and facetectomies in order to adequately alleviate foraminal stenosis and to obtain adequate disc space distraction. More extensive releases result in greater instability often requiring additional instrumentation (ie, bilateral interbody cages); prolonged operative times have been noted in several studies using this bilateral technique. ${ }^{35,36}$ Additionally, Pan et $\mathrm{al}^{35}$ reported that the bilateral technique was associated with increased intraoperative blood loss. Lastly, it has been well established that the MIS TLIF is associated with a significant procedural learning curve. ${ }^{14-17}$ As the surgeon becomes more comfortable with the MIS technique, the prolonged operative time and EBL may normalize. ${ }^{35}$ While there are many reasons for the observed increase in operative time, the clinical significance of this finding remains poorly understood as LOS, periop-

Table 4. Outcomes of subgroup analyses. ${ }^{\mathrm{a}}$

\begin{tabular}{|c|c|c|c|c|c|c|}
\hline & \multicolumn{2}{|c|}{ Grade I Spondylolisthesis ${ }^{\text {b }}$} & \multirow[b]{2}{*}{$P$ Value ${ }^{c}$} & \multicolumn{2}{|c|}{ Grade II Spondylolisthesis $^{b}$} & \multirow[b]{2}{*}{$P$ Value } \\
\hline & Isthmic $(\mathrm{N}=22)$ & Degenerative $(N=149)$ & & Isthmic $(N=40)$ & Degenerative $(\mathrm{N}=12)$ & \\
\hline Operative time, mean $\pm \mathrm{SD}$, min & $146.3 \pm 57.2$ & $121.8 \pm 40.3$ & .030 & $146.7 \pm 35.9$ & $133.6 \pm 49.8$ & .011 \\
\hline Estimated blood loss, $\mathrm{mL}$ & $69.8 \pm 42.9$ & $66.4 \pm 55.0$ & .832 & $113.5 \pm 123.0$ & $85.4 \pm 101.9$ & .076 \\
\hline Length of hospital stay, h & $67.0 \pm 32.2$ & $68.5 \pm 45.0$ & .861 & $69.3 \pm 61.7$ & $85.0 \pm 53.2$ & .719 \\
\hline \multicolumn{7}{|l|}{$\mathrm{VAS}$, mean $\pm \mathrm{SD}$} \\
\hline 6-wk VAS & $4.2 \pm 2.0$ & $3.6 \pm 2.2$ & .640 & $4.2 \pm 2.3$ & $2.6 \pm 2.2$ & .560 \\
\hline 12-wk VAS & $3.5 \pm 1.9$ & $3.5 \pm 2.3$ & .456 & $3.8 \pm 2.1$ & $2.2 \pm 2.5$ & .395 \\
\hline 6-mo VAS & $3.8 \pm 2.3$ & $3.2 \pm 2.5$ & .569 & $4.0 \pm 2.7$ & $1.7 \pm 1.4$ & .758 \\
\hline \multicolumn{7}{|l|}{ Change in VAS, mean $\pm \mathrm{SD}^{\mathrm{d}}$} \\
\hline$\triangle \mathrm{VAS}$ at $6 \mathrm{wk}$ & $-2.0 \pm 2.5$ & $-3.2 \pm 3.0$ & .831 & $-2.8 \pm 3.1$ & $-2.3 \pm 4.0$ & .876 \\
\hline$\Delta \mathrm{VAS}$ at $12 \mathrm{wk}$ & $-3.4 \pm 3.7$ & $-3.5 \pm 2.8$ & .456 & $-3.8 \pm 3.1$ & $-2.7 \pm 5.0$ & .395 \\
\hline$\Delta \mathrm{VAS}$ at $6 \mathrm{mo}$ & $-4.1 \pm 3.4$ & $-4.0 \pm 3.1$ & .113 & $-4.1 \pm 3.1$ & $-3.7 \pm 4.3$ & .791 \\
\hline Complications, $\%(n)^{\mathrm{e}}$ & $0.0(0)$ & $2.7(4)$ & $-{ }^{\mathrm{f}}$ & $2.5(1)$ & $0.0(0)$ & $-{ }^{\mathrm{f}}$ \\
\hline Arthrodesis at $1 \mathrm{y}, \%$ (n) & 86.4 (19) & $97.3(145)$ & .663 & $90.0(36)$ & $100.0(12)$ & .503 \\
\hline Revision, $\%(\mathrm{n})^{\mathrm{g}}$ & $13.6(3)$ & $8.1(12)$ & .280 & $7.5(3)$ & $8.3(1)$ & $-{ }^{f}$ \\
\hline
\end{tabular}

Abbreviations: SD, standard deviation; VAS, visual analogue scale.

${ }^{a}$ Boldface indicates statistical significance.

${ }^{\mathrm{b}}$ Three patients were excluded due to having both isthmic and degenerative spondylolisthesis.

${ }^{\mathrm{c}} P$ value is from Poisson regression with robust error variance (binary outcomes) or linear regression (continuous outcomes) adjusted for age, sex, body mass index, operative level (L5-S1 versus other), smoking status, comorbidity burden, and preoperative visual analogue scale.

${ }^{\mathrm{d}}$ Change in VAS (for each patient) = postoperative VAS $(6 \mathrm{wk}, 12 \mathrm{wk}, 6 \mathrm{mo})$ - preoperative VAS.

${ }^{e}$ Complications include pseudo bowel obstruction requiring rectal tube placement, epidural hematoma/fluid collection requiring irrigation and debridement (3), thromboembolic event (deep vein thrombosis [2])

${ }^{\mathrm{f}}$ Statistical analysis was not performed for variables with a total of $\leq 5$ occurrences

${ }^{\mathrm{g}}$ Revisions include pseudarthrosis (11), failed spinal instrumentation, recurrent symptoms requiring revision laminectomy (4), epidural hematoma/fluid collection requiring irrigation and debridement (3), recurrent stenosis with radiculopathy and foot drop 
erative pain scores, and arthrodesis, complication, and revision rates were similar between cohorts.

The present study supports the results of several others. Before the adaptation of modern MIS techniques for TLIF, Lauber et $\mathrm{al}^{37}$ performed a prospective study comparing the clinical and radiographic outcomes between low-grade IS and DS after a traditional open TLIF. The authors reported comparable initial improvement in Oswestery Disability Index (ODI), VAS pain scores, and shortterm radiographic outcomes at all follow-up visits before 2 years. The authors demonstrated a cumulative $94.8 \%$ arthrodesis rate and concluded that open TLIF was a safe and effective method to treat low-grade IS and DS.

In 2010, Wang et $\mathrm{al}^{27}$ performed a prospective analysis of clinical and radiographic factors between 39 IS and 46 DS cases after MIS and open TLIF. The authors observed no significant difference in operative time, VAS, or ODI preoperatively and at final follow up. However, the MIS cohort experienced reduced EBL, total blood transfusions, LOS, and pain in the immediate postoperative period as demonstrated by a reduced VAS score. The authors concluded that MIS TLIF demonstrated superior outcomes for low-grade IS and DS populations compared to the open cohort. Their investigation was limited, however, by the lack of stratification into grades I and II spondylolisthesis.

In a 2015 study performed by Kim et $\mathrm{al}^{26}$ the authors compared clinical and radiographic outcomes between low-grade IS and DS after MIS TLIF in 41 patients (18 IS and 23 DS). The authors used a unilateral approach with single interbody cage placement and bilateral instrumentation resulting in similar clinical outcomes between cohorts. In contrast to the current study, the authors observed no difference in operative time between cohorts, as well as reduced arthrodesis rates in the DS cohort. The authors also reported VAS and ODI pain scores, and perioperative and postoperative clinical outcomes were no different between cohorts; however, of the radiographic parameters measured, the IS cohort demonstrated a greater restoration of disc height compared to the DS cohort. The authors concluded that MIS TLIF was an effective treatment option for low-grade IS and DS. However, the study was severely limited by the underpowered patient population. Only 2 of the 41 total patients were diagnosed with grade II spondylolisthesis. ${ }^{26}$ Based on the limited scope of pathology examined in the study of Kim et al, the current study aimed to analyze the outcomes between grade II IS and DS after MIS TLIF, endorsing the successful application of MIS TLIF for all low-grade patients.

Among the 223 patients included in the current study, $62(27.8 \%)$ were diagnosed with IS and 161 $(72.2 \%)$ were diagnosed with DS. To the best of the authors' knowledge, this is the largest study comparing low-grade IS and DS after MIS TLIF. The younger age, lower comorbidity burden, and difference in location of spondylolisthesis experienced by the IS compared to the DS cohort is intuitive and can be explained by the pathologic variation and age of presentation. IS and DS most commonly present in healthy adolescents at L5-S1, and in elderly women at L4-L5, respectively. ${ }^{1,3,4}$ The differences in age and location of spondylolisthesis observed between cohorts are therefore characteristic of their pathologic processes. Similarly, the age difference between cohorts explains the significant difference in comorbidity burden, as an increase in age is associated with increasing prevalence, number, and severity of comorbid conditions. ${ }^{38}$

This study has several limitations. Although different procedural techniques may have been used for each spondylolisthesis grade, a single surgeon performed them. This ultimately cut down on the operative variability within patients with the same diagnosis. Additionally, the consistency between operative outcomes suggests the successful application of both techniques among the patient population examined. However, the procedures were used on patients with varying pathologic difficulty, necessitating a subgroup analysis among spondylolisthesis grades. Secondly, the techniques described were all performed by a single surgeon at a single academic institution, limiting the generalizability. Finally, the study was performed retrospectively, introducing possible selection and information bias.

The results of this study demonstrate that MIS TLIF is an effective treatment option for both grade I and II DS and IS patients, despite the reduced stability inherent with IS. Although principally the senior surgeon's preferred technique, using a bilateral approach and bilateral interbody cages safely and effectively treated grade II IS with equivalent clinical outcomes compared to a unilateral approach and single interbody cage for grade I IS and DS and grade II DS. Future studies prospectively assessing additional risk factors as well as 
detailed radiographic analyses, including lordotic angle and subsidence rate, will allow for more accurate determination of the best candidates for MIS TLIF as the treatment for IS.

\section{REFERENCES}

1. Schulte TL, Ringel F, Quante M, Eicker SO, MucheBorowski C, Kothe R. Surgery for adult spondylolisthesis: a systematic review of the evidence. Eur Spine J. 2015; 25(8):2359-2367.

2. Kirkaldy-Willis WH, Farfan HF. Instability of the lumbar spine. Clin Orthop Relat Res. 1982;165:110-123.

3. Omidi-Kashani F, Hasankhani EG, Rahimi MD, Khanzadeh R. Comparison of functional outcomes following surgical decompression and posterolateral instrumented fusion in single level low grade lumbar degenerative versus isthmic spondylolisthesis. Clin Orthop Surg. 2014;6(2):185-189.

4. Sulaiman WA, Singh M. Minimally invasive versus open transforaminal lumbar interbody fusion for degenerative spondylolisthesis grades 1-2: patient-reported clinical outcomes and cost-utility analysis. Ochsner J. 2014;14(1):32-37.

5. Wiltse LL, Winter RB. Terminology and measurement of spondylolisthesis. J Bone Joint Surg Am. 1983;65(6):768-772.

6. Bouras T, Korovessis P. Management of spondylolysis and low-grade spondylolisthesis in fine athletes. A comprehensive review. Eur J Orthop Surg Traumatol. 2015;25(Suppl 1):S167-S175.

7. Palepu V, Kodigudla M, Goel VK. Biomechanics of disc degeneration. Adv Orthop. 2012;2012:726210.

8. Jaumard NV, Welch WC, Winkelstein BA. Spinal facet joint biomechanics and mechanotransduction in normal, injury and degenerative conditions. J Biomech Eng. 2011;133(7):071010.

9. Schnake KJ, Putzier M, Haas NP, Kandziora F. Mechanical concepts for disc regeneration. Eur Spine J. 2006;15(Suppl 3):S354-S360.

10. Li W, Wang S, Xia Q, et al. Lumbar facet joint motion in patients with degenerative disc disease at affected and adjacent levels: an in vivo biomechanical study. Spine. 2011;36(10):E629-E637.

11. Oppenheimer JH, DeCastro I, McDonnell DE. Minimally invasive spine technology and minimally invasive spine surgery: a historical review. Neurosurg Focus. 2009;27(3):E9.

12. Skovrlj B, Gilligan J, Cutler HS, Qureshi SA. Minimally invasive procedures on the lumbar spine. World $J$ Clin Cases. 2015;3(1):1-9.

13. Barbagallo GM, Yoder E, Dettori JR, Albanese V. Percutaneous minimally invasive versus open spine surgery in the treatment of fractures of the thoracolumbar junction: a comparative effectiveness review. Evid Based Spine Care J. 2012;3(3):43-49.

14. Lee JC, Jang HD, Shin BJ. Learning curve and clinical outcomes of minimally invasive transforaminal lumbar interbody fusion: our experience in 86 consecutive cases. Spine. 2012;37(18):1548-1557.

15. Silva PS, Pereira P, Monteiro P, Silva PA, Vaz R. Learning curve and complications of minimally invasive transforaminal lumbar interbody fusion. Neurosurg Focus. 2013;35(2):E7.
16. Lee KH, Yeo W, Soeharno H, Yue WM. Learning curve of a complex surgical technique: minimally invasive transforaminal lumbar interbody fusion (MIS TLIF). J Spinal Disord Tech. 2014;27(7):E234-E240.

17. Nandyala SV, Fineberg SJ, Pelton M, Singh K. Minimally invasive transforaminal lumbar interbody fusion: one surgeon's learning curve. Spine J. 2014;14(8):1460-1465.

18. Ahn J, Tabaraee E, Singh K. Minimally invasive transforaminal lumbar interbody fusion. J Spinal Disord Tech. 2015;28(6):222-225.

19. Phan K, Rao PJ, Kam AC, Mobbs RJ. Minimally invasive versus open transforaminal lumbar interbody fusion for treatment of degenerative lumbar disease: systematic review and meta-analysis. Eur Spine J. 2015;24(5):1017-1030.

20. Khan NR, Clark AJ, Lee SL, Venable GT, Rossi NB, Foley KT. Surgical outcomes for minimally invasive vs open transforaminal lumbar interbody fusion: an updated systematic review and meta-analysis. Neurosurgery. 2015;77(6):847-874.

21. Adogwa O, Parker SL, Bydon A, Cheng J, McGirt MJ. Comparative effectiveness of minimally invasive versus open transforaminal lumbar interbody fusion: 2-year assessment of narcotic use, return to work, disability, and quality of life. $J$ Spinal Disord Tech. 2011;24(8):479-484.

22. Crandall DG, Revella J, Patterson J, Huish E, Chang M, McLemore R. Transforaminal lumbar interbody fusion with rhBMP-2 in spinal deformity, spondylolisthesis, and degenerative disease - part 1: large series diagnosis related outcomes and complications with 2- to 9-year follow-up. Spine. 2013;38(13):1128-1136.

23. Crandall DG, Revella J, Patterson J, Huish E, Chang M, McLemore R. Transforaminal lumbar interbody fusion with rhBMP-2 in spinal deformity, spondylolisthesis, and degenerative disease - part 2: BMP dosage-related complications and long-term outcomes in 509 patients. Spine. 2013;38(13):11371145 .

24. Aoki Y, Yamagata M, Ikeda Y, et al. A prospective randomized controlled study comparing transforaminal lumbar interbody fusion techniques for degenerative spondylolisthesis: unilateral pedicle screw and 1 cage versus bilateral pedicle screws and 2 cages. J Neurosurg Spine. 2012;17(2):153-159.

25. Adogwa O, Parker SL, Davis BJ, et al. Cost-effectiveness of transforaminal lumbar interbody fusion for grade I degenerative spondylolisthesis. J Neurosurg Spine. 2011;15(2):138-143.

26. Kim JY, Park JY, Kim KH, et al. Minimally invasive transforaminal lumbar interbody fusion for spondylolisthesis: comparison between isthmic and degenerative spondylolisthesis. World Neurosurg. 2015;84(5):1284-1293.

27. Wang J, Zhou Y, Zhang ZF, Li CQ, Zheng WJ, Liu J. Comparison of one-level minimally invasive and open transforaminal lumbar interbody fusion in degenerative and isthmic spondylolisthesis grades 1 and 2. Eur Spine J. 2010;19(10):17801784 .

28. Kim R, Singla A, Samdani AF. Classification of spondylolisthesis. In: Wollowick AL, Sarwahi V, eds. Spondylolisthesis: Diagnosis, Non-Surgical Management, and Surgical Techniques. New York: Springer; 2015:95-106.

29. Zou G. A modified Poisson regression approach to prospective studies with binary data. Am J Epidemiol. 2004;159(7):702-706.

30. Liu X, Li G, Wang J, Zhang H. Minimally invasive 
unilateral vs. bilateral pedicle screw fixation and lumbar interbody fusion in treatment of multi-segment lumbar degenerative disorders. Med Sci Monit. 2015;21:3652-3657.

31. Elboghdady IM, Naqvi A, Jorgenson AY, MarquezLara A, Singh K. Minimally invasive transforaminal lumbar interbody fusion for lumbar spondylolisthesis. Ann Transl Med. 2014;2(10):99.

32. Eliades P, Rahal JP, Herrick DB, et al. Unilateral pedicle screw fixation is associated with reduced cost and similar outcomes in selected patients undergoing minimally invasive transforaminal lumbar interbody fusion for L4-5 degenerative spondylolisthesis. Cureus. 2015;7(2):e249.

33. Osterman K, Schlenzka D, Poussa M, Seitsalo S, Virta L. Isthmic spondylolisthesis in symptomatic and asymptomatic subjects, epidemiology, and natural history with special reference to disk abnormality and mode of treatment. Clin Orthop Relat Res. 1993;297:65-70.

34. Scheer JK, Auffinger B, Wong RH, et al. Minimally invasive transforaminal lumbar interbody fusion (TLIF) for spondylolisthesis in 282 patients: in situ arthrodesis versus reduction. World Neurosurg. 2015;84(1):108-113.

35. Pan J, Li L, Qian L, et al. Spontaneous slip reduction of low-grade isthmic spondylolisthesis following circumferential release via bilateral minimally invasive transforaminal lumbar interbody fusion: technical note and short-term outcome. Spine. 2011;36(4):283-289.

36. Quraishi NA, Rampersaud YR. Minimal access bilateral transforaminal lumbar interbody fusion for high-grade isthmic spondylolisthesis. Eur Spine J. 2013;22(8):1707-1713.

37. Lauber S, Schulte TL, Liljenqvist U, Halm H, Hackenberg L. Clinical and radiologic 2-4-year results of transforaminal lumbar interbody fusion in degenerative and isthmic spondylolisthesis grades 1 and 2. Spine. 2006;31(15):1693-1698.

38. Piccirillo JF, Vlahiotis A, Barrett LB, Flood KL, Spitznagel EL, Steyerberg EW. The changing prevalence of comorbidity across the age spectrum. Crit Rev Oncol Hematol. 2008;67(2):124-132.

Disclosures and COI: No funds were received in support of this work. No benefits in any form have been or will be received from any commercial party related directly or indirectly to the subject of this manuscript. No Food and Drug Administration device or drug status to report. We will not discuss unlabeled or investigational uses of any commercial product or device.

Corresponding Author: Kern Singh, MD, Department of Orthopaedic Surgery, Rush University Medical Center, 1611 W. Harrison St, Suite \#300, Chicago, IL 60612. Phone: (312) 432-2373; Fax: (708) 492-5373; E-mail: kern.singh@ rushortho.com.

Published 30 April 2020

This manuscript is generously published free of charge by ISASS, the International Society for the Advancement of Spine Surgery. Copyright (c) 2020 ISASS. To see more or order reprints or permissions, see http://ijssurgery.com. 